\title{
Field Team Syndromic Surveillance for Mass Gatherings: NCAA Final Four 2017
}

\author{
William E. Smith*1, Rebecca Scranton², Melissa Kretschmer ${ }^{1}$, Karen Zabel ${ }^{1}$ and \\ Kristen Pogreba-Brown ${ }^{3}$
}

${ }^{1}$ Office of Epidemiology, Maricopa County Department of Public Health, Phoenix, AZ, USA; ${ }^{2}$ Arizona Department of Health Services, Phoenix, AZ, USA; ${ }^{3}$ University of Arizona, College of Public Health, Phoenix, AZ, USA

\section{Objective}

To describe and present results of field-based near-real time syndromic surveillance conducted at first aid stations during the 2017 National Collegiate Athletic Association Division I Men's College Basketball Championship (Final Four) events, and the use of field team data to improve situational awareness for Mass Gathering events.

\section{Introduction}

Final Four-associated events culminated in four days of intense activity from $3 / 31 / 17-4 / 3 / 17$, which attracted an estimated 400,000 visitors to Maricopa County (population 4.2 million). Field teams of staff and volunteers were deployed to three days of Music Fest, four days of Fan Fest, and three Final Four games (Games) as part of an enhanced epidemiologic surveillance system.

\section{Methods}

Attendees presenting to first aid stations were requested to complete an electronic questionnaire which captured illness and injury syndromes (after needed care was given). Emergency Medical Services technicians and nurses (EMS) conducted patient care. These were submitted and epidemiologically assessed in near-real-time to rapidly identify threats. Syndrome-specific data were mapped during events to identify spatial clustering. Field Teams were provided with case contact log sheets, suspicious substance investigation and exposure registry forms to allow rapid investigation of significant public health events. Patient Presentation Rates (PPR) and Transport to Hospital Rates (TTHR) were calculated per 10,000 attendees. Patients presenting per hour of event and transports per hour of event were calculated. Field reports were included in daily reports to inter-disciplinary partners, and shared during regular Multi-Agency Coordination Center briefings.

\section{Results}

301 field questionnaires were completed, including 146 from Final Four games (Games), 127 from the Music Fest, and 28 from the Fan Fest (see Figure).

Among the 153,780 attendees of the three Games, there were 146 cases who presented to one of five first aid stations (over 12 hours). There were 27 illness cases who sought care (18.5\% of Games cases), among whom $21(78 \%)$ were assessed by EMS. Illness cases not assessed by EMS $(\mathrm{n}=6)$ included mostly allergy symptoms/medication needs. There were 50 injury cases who sought care (34.2\% of Games cases), among whom 10 (20\%) were assessed by EMS. Sixty (41.1\%) persons presented seeking a pain reliever, and $9(6.2 \%)$ presented seeking an antacid. Games experienced a PPR of 9.5, and a TTHR of 0.52 . Patients presented at 12.2 per hour on average and there were eight transports to medical facilities ( 0.66 per hour).

There were 127 cases among an estimated 135,000 Music Fest attendees who presented to one of two first aid stations (and at times 2 roving teams) over 3 days ( 22.5 hours) from $3 / 31$ to $4 / 2$. Illnesses accounted for 29 cases (22.8\% of Music Fest cases) and 28 of 29 were assessed by EMS. There were 68 injury cases who sought care
(53.5\% of Music Fest cases), among whom 22 (32.4\%) were assessed by EMS. Twenty-seven persons $(21.3 \%)$ presented seeking a pain reliever and $2(1.6 \%)$ sought an antacid. Music Fest results included a PPR of 9.4, and a TTHR of 0.15 . There were 5.6 patients presenting per hour on average, and there were two transports to the hospital (0.09 per hour).

At the Fan Fest there were 28 cases among an estimated 50,803 attendees presenting to the first aid station (or roving teams) from $3 / 31-4 / 3$ (over 37 hours). Most cases sought care for an injury $(n=22$, $78.6 \%$ of cases). Four persons sought care for an illness (14.3\%), all with relatively minor complaints. For the Fan Fest, there was a PPR of 5.5 , and a TTHR of 0 (there were no transports to the hospital). There were 0.76 patient presentations per hour on average.

No geographic clustering or public health threats requiring investigation were identified at any of the three sites. Interdisciplinary partners requested additional field data during the response.

\section{Conclusions}

Injuries were more common than illnesses at all three sites. Visits requiring pain relievers only were more common at Games $(41.1 \%)$ than at Music Fest $(21.3 \%)$ or Fan Fest (3.6\%).

A greater percentage of visits requiring EMS assessment were seen at the Fan Fest (78.6\%) than at the Music Fest (40.2\%) or the Games $(21.2 \%)$

The PPRs per 10,000 attendees were highest at the Games (9.5) and Music Fest (9.4), compared to the Fan Fest (5.5). The TTHR per 10,000 attendees was highest at the Games (0.52), compared to the Music Fest (0.15) and Fan Fest (0.0). The Music Fest field team reported greater effectiveness at fixed first aid stations compared to traveling with roving EMS teams. Field reports enhanced health and medical situational awareness and information sharing as evidenced by requests from interdisciplinary partners for additional field data.

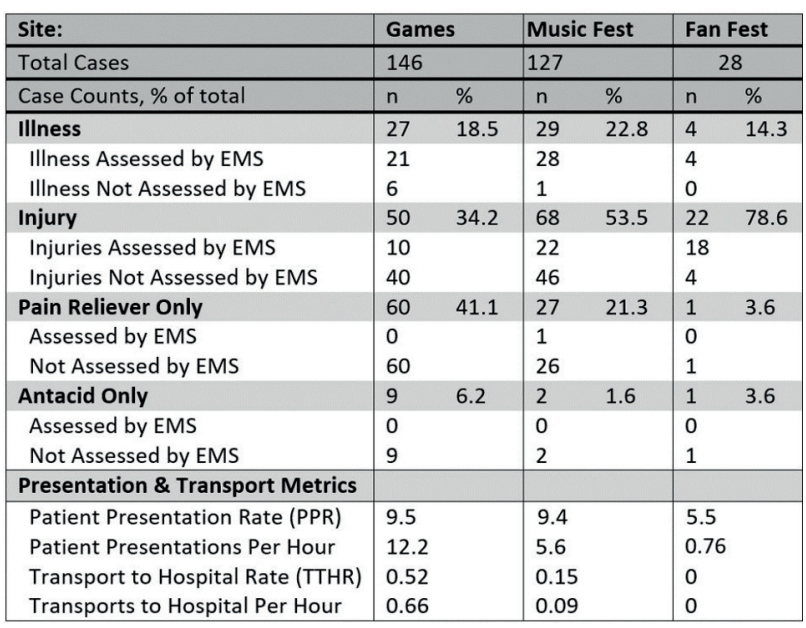

Figure: Syndromes and Patient Presentation Metrics at Games, Music Fest and Fan Fest. 


\section{ISDS 2018 Conference Abstracts}

\section{Keywords}

Mass Gathering; syndromic surveillance; epidemiological surveillance; public health surveillance; Final Four

\section{Acknowledgments}

Thank you to the 2017 Final Four Enhanced Surveillance Field Teams

\section{*William E. Smith}

E-mail: williamsmith@mail.maricopa.gov 